\title{
La conférence des directeurs médicaux de la Suisse latine
}

\section{Bernard Vermeulen}

Président $^{*}$

* Au nom des membres de la conférence des directeurs médicaux de la Suisse latine

1 Maréchal JP. Réformer l'hôpital. Direction médicale pivot de la réorganisation. Paris: Hermes Sciences Publicat; 1996.

Correspondance: Prof. B. Vermeulen Directeur médical hôpital fribourgeois Case postale $\mathrm{CH}-1708$ Fribourg Tél. 0264268839

vermeulenb@h-fr.ch
Au cours des années 2008 et 2009, les directeurs médicaux d'hôpitaux de la Suisse latine (Centre Hospitalier Universitaire Vaudois, Ente Ospedaliero Cantonale, hôpital fribourgeois, Hôpital neuchâtelois, Hôpital du Jura, Hôpitaux Universitaires de Genève, Réseau santé Valais, Etablissements Hospitaliers du Nord Vaudois) se sont rencontrés pour échanger et débattre de sujets touchant leurs activités et responsabilités. Ce groupe a souhaité formaliser son existence sous forme d'une «conférence des directeurs médicaux de la Suisse latine» et a établi un cahier des charges et un règlement de fonctionnement.

Quel est le rôle d'un directeur médical? Cette question revient souvent chez nos collègues cliniciens, sous entendant une alliance contre nature avec les métiers de l'administration. J.-P. Maréchal a tenté de formuler une réponse, il y a 13 ans déjà, dans son livre intitulé «Réformer l'hôpital» [1]. Il écrit: L'hôpital constitue, aujourd'hui, l'un des axes prioritaires de réflexion sur la nécessaire réforme de notre système de santé. Il ne s'agit pas seulement de produire des services à coût maîtrisé, mais également de mieux répondre à l'aspiration légitime des patients à des soins de meilleure qualité. J.-P. Maréchal essaye de montrer que l'intégration d'une sensibilité médicale dans la direction générale crée un dynamisme qui permet de trouver un nouvel équilibre relationnel entre les acteurs dirigeants de l'activité hospitalière (conseil de direction, collège des médecins, conseil d'administration), ce qui facilite à terme l'évolution des structures hospitalières.

Si nous considérons les compétences attendues d'un directeur médical, nous pouvons identifier six ensembles cohérents relevant chacun d'une expertise particulière: gestion organisationnelle et opérationnelle du corps médical, ressources humaines médicales, information médicale, qualité médicale, hygiène hospitalière et gestion administrative.

Certes, il ne s'agit pas de reprendre à son compte toutes ces activités, mais il doit être présent pour coordonner l'action et le travail des comités consultatifs et/ou d'expertise afin de permettre à ses collègues d'être des partenaires actifs. Il doit veiller à ce que les décisions de la direction générale ou du conseil d'administration soient compatibles avec les objectifs thérapeutiques et les moyens nécessaires à leur réalisation.

Les établissements universitaires constatent déjà qu'un seul directeur médical ne suffit plus pour faire face aux exigences de gestion qui lui sont demandées. Faut-il progressivement créer un staff de la direction médicale ou mettre des forces administratives à disposition des médecins chefs de service? Une solution ne sera pas trouvée de si tôt et relève des particularismes des différentes structures de gestion hospitalière.

Reste que dans l'actuel contexte économique tendu, accompagné d'un développement effréné des procédures juridiques, un fossé est en train de se creuser entre les vœux de l'administration et la disponibilité et capacité du terrain. La difficile tâche des directeurs médicaux consiste alors à trouver un juste équilibre entre les exigences et les moyens limités à disposition. C'est à cette fin qu'ils ont décidé d'unir leurs expériences pour coordonner leurs activités.

\section{Le champ d'action de la conférence des directeurs} médicaux de la Suisse latine a été formalisé au moyen d'un cahier des charges qui est le suivant:

- faciliter les relations inter-établissements;

- donner un avis sur les projets d'ordre politique qui concernent le fonctionnement des établissements médicaux;

- harmoniser l'organisation de l'offre médicale;

- coordonner les procédures de qualité et d'évaluation de la pratique professionnelle;

- favoriser l'échange de compétences des professionnels médicaux;

- favoriser et coordonner les processus de formation post-gradué;

- coordonner l'utilisation de référentiels;

- favoriser le développement de la recherche médicale et la participation à des études multicentriques;

- promouvoir et veiller au respect des principes éthiques et de déontologie en vigueur;

- être des interlocuteurs privilégiés pour les autorités politiques;

- organiser la relève pour les postes cadres de nos hôpitaux.

La réforme des missions des hôpitaux n'est pas qu'une question de médecine intra-hospitalière. Elle a une implication politique, économique et sociétale. Les représentants du corps médical, et plus particulièrement les directeurs médicaux, se doivent d'être les porteparoles de la sensibilité médicale dans ce débat, tâche qui s'effectue encore mieux en commun. 